\title{
MODEL CALCULATIONS OF THE PHOTOSPHERIC LAYERS OF SOLAR MAGNETIC FLUXTUBES
}

\author{
OSKAR STEINER and J.O. STENFLO \\ Institut für Astronomie \\ ETH-Zentrum \\ 8092 Zürich \\ Switzerland
}

\begin{abstract}
Multi-dimensional radiative energy transport is coupled self-consistently to magnetohydrostatic solutions for fluxtubes with rotational symmetry. It is shown that the photospheric layers of plage and network fluxtubes are heated by radiation by as much as $300 \mathrm{~K}$ at equal geometrical height. The amount of heating depends on the density reduction within the tube. The results are compared with observational data and the most recent semi-empirical model.
\end{abstract}

\section{Magnetohydrostatics and Radiative Transport}

Magnetohydrostatic solutions of rotationally symmetric, vertically oriented magnetic fluxtubes in the solar photosphere are considered. The models should account for the basic physical processes of the tiny photospheric structures, which appear as bright points in the continuous spectrum of plages and network regions, and which are observed to have strong magnetic fields in the 1-2 kG range (Stenflo, 1973, 1989).

The magnetohydrostatic equation,

$$
-\nabla p+\rho \mathbf{g}+\frac{1}{4 \pi}(\nabla \times \mathbf{B}) \times \mathbf{B}=0
$$

is solved using a finite difference scheme, allowing for fluxtubes having a sharp boundary (current sheet) where the magnetic field strength jumps from a finite value to zero. The method for solving (1) has been described in great detail in Steiner et al. (1986), who also provide an accuracy test.

As a next step, to obtain more realistic models for plage and network magnetic fluxtubes and to reduce the number of free parameters, we include an energy equation, which allows us to derive the temperature structures of such regions. We concentrate on the photospheric layers, since this is the height region most accessible to Stokes polarimetry. In this region radiative transfer is the dominant mode of energy transport. The radiative transfer equation is solved using cylinder coordinates under the constraint of radiative equilibrium:

$$
\begin{aligned}
& (\mathbf{n} \cdot \nabla) \mathrm{I}(\mathbf{r}, \mathbf{n}, \nu)=\eta(\mathbf{r}, \nu)-\chi(\mathbf{r}, \nu) \mathrm{I}(\mathbf{r}, \mathbf{n}, \nu) \\
& \int_{0}^{\infty} \chi(\mathbf{r}, \nu) \mathrm{S}(\mathbf{r}, \nu) \mathrm{d} \nu=\int_{0}^{\infty} \chi(\mathbf{r}, \nu) \mathrm{J}(\mathbf{r}, \nu) \mathrm{d} \nu .
\end{aligned}
$$

LTE is assumed with allowance for continuum scattering. The continuum opacities, scattering coefficients, and the mean molecular weight are computed using the statistical equilibrium and 
opacity code of Gustafsson (1973). Line blanketing is taken into account using opacity distribution functions (ODF) of Kurucz (1979). Couples of ODF's have been merged into one to save computer CPU-time. To avoid the inversion of the complete $\boldsymbol{\Lambda}$-matrix the formal solution of the transfer equation

$$
J_{\nu}(\mathbf{r})=\mathbf{\Lambda}_{\nu}\left(\mathbf{r}, \mathbf{r}^{\prime}\right) \mathrm{B}_{\nu}\left(\mathbf{r}^{\prime}\right)
$$

is only computed, using an accelerated $\lambda$-iteration to obtain iterative temperature corrections. This procedure is based on an operator perturbation technique as will be described in Steiner (1989). The method provides fast convergence also in high opacity atmospheres. This is greatly needed since radiative transfer within the tube may still be important even far below the $\tau=1$ level, due to the inhibition of convective energy transport by the magnetic field.

The formal solution of the radiative transfer equation in cylinder coordinates has been computed using a modified version of the program CYL2D written by P. Kunasz, which is based on the short characteristic method of Kunasz and Auer (1988). This code reduces the 3D-transfer problem to an integration in several $2 \mathrm{D}$-planes by exploiting the rotational symmetry.
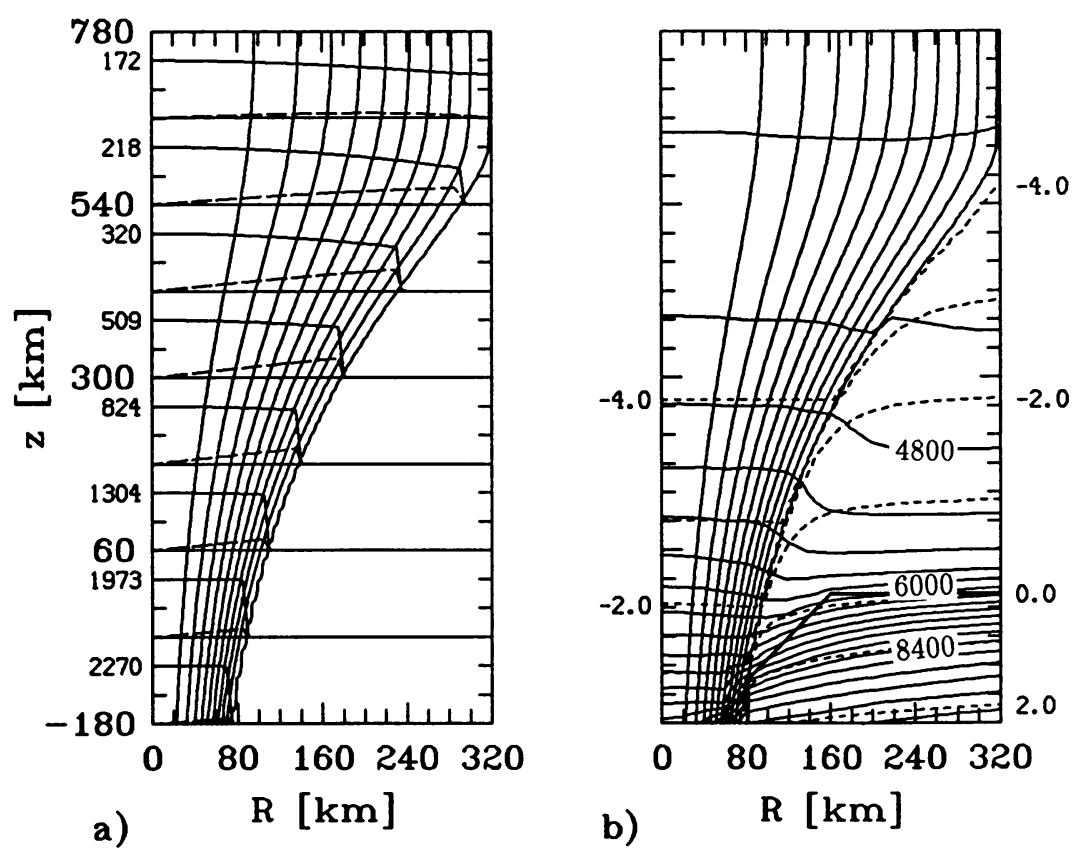

Figure 1. Model magnetic fluxtube representative of examples considered in the text. a) Field lines delineating the fluxtube which is separated from the surrounding field free plasma by a thin current sheet. Superimposed on the figure are horizontal curves showing the radial variation of $B_{z}$ (solid lines) and $B_{r}$ (dashed lines), each normalized to $B_{z}$ at the axis, the value of which in Gauss is indicated to the left of each curve. b) Same fluxtube as in a) with contour lines of constant temperature (solid lines) and constant optical depth (dashed lines). $\log \tau_{5000}$ is indicated to the left and right of the figure. The region below the heavy solid line is convectively unstable and its temperature has been prescribed (see text). 


\section{Magnetic Field and Temperature Structure}

Fig. 1a shows representative magnetic field lines of a fluxtube with a radius of $100 \mathrm{~km}$ and a field strength of about $1600 \mathrm{G}$, at $z=0$ (which refers to the height $z$ at which $\tau_{5000}=1$ in the undisturbed atmosphere ${ }^{1}$ ). Included in the figure are curves giving the field strength as a function of radius at several height levels. The field of the $z$ component decreases from the fluxtube axis to the fluxtube boundary as a consequence of magnetic tension forces (Steiner and Pizzo, 1989). Because of the decreasing gas pressure the fluxtubes expand strongly with height and finally merge with neighbouring tubes into a uniform vertical weak field. The effect of the neighbouring fluxtubes is accounted for in the model by the choice of the boundary conditions for Equation (1).

Fig. 1b shows the temperature structure of the fluxtube of Fig. 1a together with iso- $\tau_{5000}$ lines. For the radiative transfer, Eq. (4), periodic boundary conditions have been used to take the influence of neighbouring fluxtubes into account. Since convective energy transport is not treated in the present model the temperature structure in the corresponding region (area below the heavy solid line in Fig. 1b) is prescribed, using the values of Grossmann-Doerth et al. (1988). The fluxtube interior is much less opaque than its surroundings because of its reduced density. It can be imagined as a hole in the solar surface through which radiation escapes more easily, causing an elevated temperature in the overlying photospheric layers.

Fig. 2a shows the variation of temperature with optical depth along the axis of fluxtubes having field strengths of 1600 (dot-dashed line) and 1500 Gauss (dashed line), as well as along the symmetry axis between the fluxtubes (solid line), which is very close to the temperature of the undisturbed atmosphere. The dotted line represents the semi-empirical model of Keller (1989). Note that the characteristic temperature depression around $\log \tau_{5000}=-2$ present in the semi-empirical model can not be explained by radiative effects. Fig. $2 b$ shows for the same models the temperature as a function of geometrical height $z$.

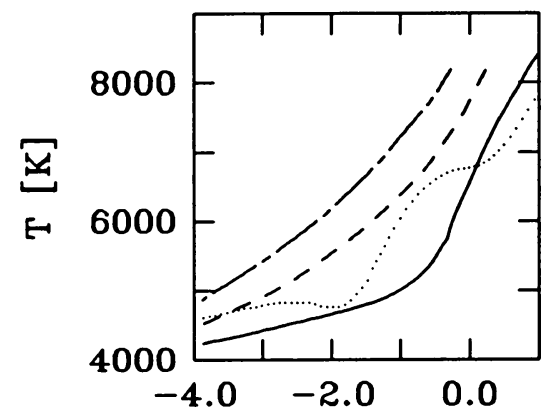

a)

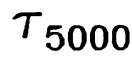

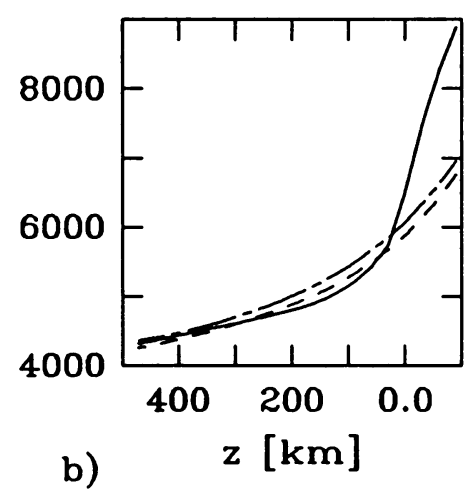

Figure 2. a) Temperature with optical depth along the symmetry axis between two neighbouring fluxtubes (solid line) and along the fluxtube axis for a tube with $B_{z}(z=0)=1600 \mathrm{G}$ (dot-dashed) and $1500 \mathrm{G}$ (dashed line). The dotted line refers to the semi-empirical model of Keller (1989). b) Temperature curves as functions of geometrical height $z$.

\footnotetext{
1 With 'undisturbed atmosphere' we mean the model atmosphere obtained with the same code as used for the fluxtube calculations but without magnetic fields. The resulting undisturbed or quiet atmosphere is very close to the solar model of Kurucz (1979).
} 


\section{Center to Limb Continuum Contrast}

The solid line in Fig. 3 shows the calculated continuum contrast $\gamma(\lambda=5000 \AA)$ for values of $\mu=\cos \theta$ close to the solar limb and for a model with $B(z=0)=1500 \mathrm{G}, R(z=0)=100 \mathrm{~km}$, and a filling factor $f=0.175$. The calculated contrast is an average over a region of about $1 \times 1$ arcseconds, simulating moderate resolution. The dot-dashed and the dashed lines are moderate resolution observations taken from Badaljan (1968) and Akimov et al. (1987), respectively. Three characteristics should be noted. Firstly, the maximum continuum contrast of the model occurs around $\mu=0.2$ in agreement with observations. Secondly, the actual value of $\gamma$ depends strongly on the filling factor. This can be readily understood if we imagine that with increasing $f$ more and more fluxtubes appear in a given resolution element making it more or less uniformly bright. It is clear that the particular density and temperature structure of the fluxtube also plays a role. Thirdly, we draw attention to the sharp increase of $\gamma$ very close to the limb, which is exclusively due to the radiative heating of the tube interior above $z \approx 0$. Increasing continuum contrasts at the extreme limb $(\mu \lessgtr 0.1)$ have been reported by several authors, for example by Akimov et al. (1987).

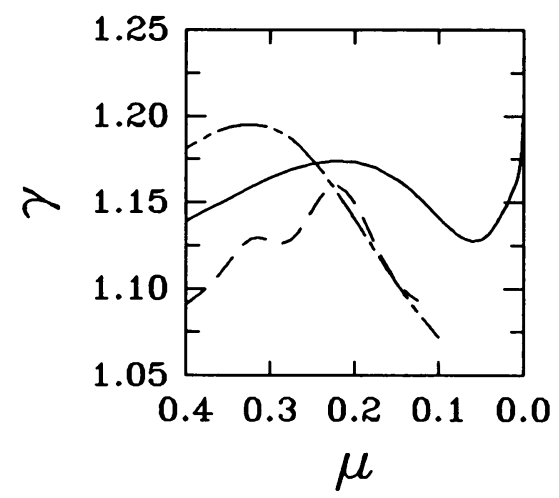

Figure 3. Center to limb variation of the continuum contrast $(\lambda=5000 \AA)$ derived from a model similar to that shown in Fig. 1 along with observed (dashed and dot-dashed lines) CLV contrasts.

ACKNOWLEDGEMENT. The authors are grateful to Dr. P. Kunasz for letting us use his yet unpublished radiative transfer code CYL2D.

\section{References}

Akimov, L.A., Belkina, I.L., Dyatel, N.P.: 1982, Astron. Zh. 59, 552

Akimov, L.A., Belkina, I.L., Dyatel, N.P., Marchenko, G.P.: 1987, Astron. Zh. 64, 126

Badaljan O.G.: 1968, Soln. Dannye. 5, 105

Grossman-Doerth, U., Knölker, M., Schüssler, M., Weisshaar, E.: 1988, in Solar and Stellar Granulation, Proc. of the OAC-NATO Advanced Research Workshop, Capri, Italy, 1988

Gustafsson, B.: 1973, Uppsala Astron. Obs. Ann. 5, No. 6

Keller, C.U.: 1989, IAU Symp. 138

Kunasz, P.B., Auer, L.H.: 1988, J. Quant. Spectrosc. Radiat. Transfer. 39, 67

Kurucz, R.L.: 1979, Astrophys. J. Suppl. Ser. 40, 1

Steiner, O.: 1989, A rapidly convergent temperature correction procedure using operator perturbation, to be submitted

Steiner, O., Pizzo, V.J.: 1989, Astron. Astrophys. 211, 447

Steiner, O., Pneuman, G.W., Stenflo, J.O.: 1986, Astron. Astrophys. 170, 126

Stenflo, J.O.: 1973, Solar Phys. 32, 41

Stenflo, J.O.: 1989, The Astron. Astrophys. Rev. 1, 3 